\title{
Expression of Concern to: Knockdown of ZFR suppresses cell proliferation and invasion of human pancreatic cancer
}

Xiaolan Zhao ${ }^{\text {**, Man Chen }}{ }^{2}$ and Jishan $\operatorname{Tan}^{3}$

\section{Expression of Concern: Biol Res (2016) 49:26} https://doi.org/10.1186/s40659-016-0086-3

Concerns have been raised about this article [1] relating to the appropriateness of the use of the shRNA (5'-GCG GAGGGTTTGAAAGAATATCTCGAGATATTCTTT CAAACCCTCCGCTTTTTT- ${ }^{\prime}$ ) as a non-targeting control and similarities in text and formatting with other published articles. This is currently under investigation and appropriate editorial action will be taken once the investigation is concluded. The authors did not respond to our correspondence regarding this expression of concern.

\section{Author details}

${ }^{1}$ Health Management Center, The First Affiliated Hospital of Third Military Medical University, No 30 Gaotanyan Street, Shapingba District, Chongqing 400038, China. ${ }^{2}$ School of Laboratory Medicine, Chengdu Medical College, Chengdu 610083, China. ${ }^{3}$ Department of Laboratory Medicine, Chengdu Military General Hospital, Chengdu 610083, China.

\author{
Publisher's Note \\ Springer Nature remains neutral with regard to jurisdictional claims in pub- \\ lished maps and institutional affiliations.
}

Received: 13 June 2018 Accepted: 13 June 2018

Published online: 22 June 2018

Reference

1. Zhao X, Chen M, Tan J. Knockdown of ZFR suppresses cell proliferation and invasion of human pancreatic cancer. Biol Res. 2016;49:26. https:// doi.org/10.1186/s40659-016-0086-3.

*Correspondence: zhaoxiaolan65@126.com

${ }^{1}$ Health Management Center, The First Affiliated Hospital of Third

Military Medical University, No 30 Gaotanyan Street, Shapingba District,

Chongqing 400038, China

Full list of author information is available at the end of the article 